\title{
Performance of dental impression materials: Benchmarking of materials and techniques by three-dimensional analysis
}

\author{
Heike RUDOLPH ${ }^{1}$, Michael R. S. GRAF ${ }^{1}$, Katharina KUHN ${ }^{1}$, Stephanie RUPF-KÖHLER ${ }^{1}$, Alfred EIRICH ${ }^{1}$, \\ Cornelia EDELMANN ${ }^{1}$, Sebastian QUAAS $^{2}$ and Ralph G. LUTHARDT ${ }^{1}$ \\ ${ }^{1}$ Department of Prosthetic Dentistry, Center of Dentistry, Ulm University, Albert-Einstein-Allee 11, 89081 Ulm, Germany \\ ${ }^{2}$ Private Practice, Salzstrasse 10, 87435 Kempten/Allgäu, Germany \\ Corresponding author, Heike RUDOLPH; E-mail: heike.rudolph@computerzaehne.de
}

\begin{abstract}
Among other factors, the precision of dental impressions is an important and determining factor for the fit of dental restorations. The aim of this study was to examine the three-dimensional (3D) precision of gypsum dies made using a range of impression techniques and materials. Ten impressions of a steel canine were fabricated for each of the 24 material-method-combinations and poured with type 4 die stone. The dies were optically digitized, aligned to the CAD model of the steel canine, and 3D differences were calculated. The results were statistically analyzed using one-way analysis of variance. Depending on material and impression technique, the mean values had a range between $+10.9 /-10.0 \mu \mathrm{m}$ (SD 2.8/2.3) and +16.5/-23.5 $\mu \mathrm{m}$ (SD 11.8/18.8). Qualitative analysis using colorcoded graphs showed a characteristic location of deviations for different impression techniques. Three-dimensional analysis provided a comprehensive picture of the achievable precision. Processing aspects and impression technique were of significant influence.
\end{abstract}

Keywords: Dental impression precision, Polyethers, Silicones, Hydrocolloids, Vinyl polysiloxanethers

\section{INTRODUCTION}

Three-dimensional (3D) computer-aided analyses can be used for a wide variety of planning, treatment, and research applications, and have been used in dentistry as early as the mid-1980s ${ }^{1}$. The gateway to the digital world is 3D data acquisition. Data can be acquired from different sources, such as non-contact optical digitizing, mechanical digitizing, or data from more complex medical imaging methods (e.g., computer tomography and magnetic resonance tomography). Such data and data captured at different times must be aligned from the relative measuring orientation into a common coordinate system before any analysis may be initiated ${ }^{1-4)}$.

Different impression materials for study models used in orthodontic treatment planning may be analyzed using cone-beam computer tomography data of the impressions ${ }^{5)}$ with sufficient precision for orthodontic means. Alternatively, the impressions can be poured and the resulting gypsum models can be optically digitized $^{6}$.

The analysis of materials for final impressions requires the use of micro-computed tomography ( $\mu$ CT) data $^{7)}$ or non-contact optical digitizing based on the projection, reflection and detection of structured light and from different light sources. The latter can be applied for the detection of small differences, as found in the comparison of implant impression techniques ${ }^{8}$, changes in gypsum materials over time ${ }^{9}$, the accuracy of impression techniques ${ }^{10)}$, or the influence of impression material type ${ }^{11)}$.

High-precision touch-probe digitizers can be used for mechanical digitizing ${ }^{12)}$ as long as the materials to be examined are not prone to wear or insufficient resistance against the contact pressure applied with the probe of the mechanical digitizer ${ }^{13)}$. Making replicas from the object to be analyzed allows for reinforcement with supporting materials, and can be used for a complete surface mapping with an electrical probe and an X-Y table ${ }^{14)}$.

Often, the accuracy of different elastomeric impression materials for final impressions (e.g., fastsetting, silicones, vinyl siloxanethers) is assessed by measuring intra- and inter-arch distances of study model ${ }^{15-17)}$ or by analyzing distances and angles of single abutments with specified geometrical dimensions ${ }^{18,19)}$. Image analysis of tooth contours allows for free-form shape analysis of the more complex geometries of prepared teeth ${ }^{20,21}$. Distance measurements of arches and abutments are also used for the analysis of factors other than the impression material itself: impression technique ${ }^{22-27)}$, impression tray ${ }^{28,29)}$, material volume ${ }^{30-32)}$, or the effect of using a surfactant prior to pouring an impression $^{33)}$.

Even when 3D digital data are used, the following analysis is often reduced to two-dimensional (2D) distance measurements ${ }^{4,34}$. This type of analysis implies a loss of information and may not account for the variability of dimensional accuracy as it relates to measurement site $^{35}$. The comparison of $2 \mathrm{D}$ and 3D accuracy of dental impression materials revealed dimensional discrepancies in the 3D analysis that were not found in the $2 \mathrm{D}$ analysis ${ }^{36)}$. The full picture of the dimensional accuracy can only be achieved with 3D analyses, which should be used for the characterization of the dimensional stability and accuracy of impression materials, especially where materials for final impressions are concerned ${ }^{36}$.

Three-dimensional in vitro analyses overcoming the 2D limitations focused on different impression 
materials $^{7,11,12,37)}$, impression techniques ${ }^{10)}$, impression trays ${ }^{34,38)}$, and aspects of implant impression accuracy $^{8,36,39,40)}$.

Because of a greater tolerance towards dimensional changes, the requirements for impression materials for diagnostic casts differ from those for final impressions. Impressions made with irreversible hydrocolloids should be poured immediately, as delayed pouring negatively influences their dimensional stability and precision ${ }^{41,42}$.

Elastomeric impression materials available for final impressions are vinyl siloxanether, vinyl polysiloxane, silicone (usually addition-curing, but also available as condensation-curing materials), and polyether. They are still the most common method of transferring information from the patient to the dental laboratory when making indirect restorations ${ }^{43}$. The most accurate elastomeric impression materials available are polyvinyl siloxanes, polyethers, and vinyl siloxanethers ${ }^{15,17,44)}$. For single teeth or small segments (up to four teeth), a clinically achievable precision of up to $\pm 40-50 \mu \mathrm{m}$ can be deduced for the mean deviations from clinical trials ${ }^{45-47)}$.

In vitro evaluations result in higher precision and mean dimensional changes below $\pm 20 \mu \mathrm{m}^{27,48)}$. In contrast to single teeth or small segments, in vitro full arch impressions made with polyether applying a 3D analysis showed a precision of $61.3 \pm 17.9 \mu \mathrm{m}^{49)}$.

By combining quantitative and qualitative information on the complete surface of a prepared tooth, limitations such as the need for an additional macroscopic evaluation of detail reproduction ${ }^{35)}$ can be overcome. The experimental setup will have to render an adequate representation of the complex preparations encountered in clinical dentistry ${ }^{36,50)}$. While $\mu$-CT data can overcome the limited accuracy of non-contact optical digitizing in narrow and deep areas such as pits, fossae or the cervical margins of teeth ${ }^{7}$, there will be confined access to appropriate tomography devices. Furthermore, hardening artifacts, which can be caused by metal pins commonly used for saw-cut master models could be an obstacle analog to the presence of metal prostheses in CT images ${ }^{51)}$. Common dental non-contact digitizing devices will be much more easily available and less expensive. They generally show sufficient accuracy ${ }^{52)}$. In combination with commercially available $3 \mathrm{D}$ analysis software, the proposed experimental setup can be rather effortlessly adapted by different research groups ${ }^{11)}$ and independently of changing staff.

Adapting a method developed by Luthardt et al. ${ }^{9}$, the experimental setup presented here aims at a $3 \mathrm{D}$ analysis allowing for a benchmarking of a wide range of different impression materials and impression techniques, thus providing a more complete threedimensional (3D) picture of the achievable quantitative and qualitative dimensional accuracy.

\section{MATERIALS AND METHODS}

The experimental set-up is based on a master model and its identical virtual CAD model (Fig. 1). From a stainless steel master model with a circular chamfer preparation (upper canine) impressions were made with a range of impression materials: condensation-curing silicone (cs), irreversible hydrocolloid (ih), polyether (pe), reversible hydrocolloid (rh), vinyl polysiloxane (vps), vinyl (poly) siloxanether (vpse); and different impression techniques: monophase (m), one-stage two-phase (o), two-phase putty and wash (t), diagnostic cast impressions (d), manual mixing (man), auto-mixing (aut) (Tables 1a and 2). Ten duplicate dies were made for each material/technique combination. All impressions and duplicate gypsum dies were made at room temperature $\left(21^{\circ} \mathrm{C}\right)$ in a dental office or dental laboratory environment respectively. These were optically digitized with a high-precision digitizing system (ODKM 97, Fraunhofer Institute for Applied Optics and Precision Engineering, Jena, Germany). The measurement uncertainty as given by the manufacturer was $\sim 8 \mu \mathrm{m}$. Measurements resulted in data sets with around 70,000 data points $( \pm 5,000)$ per die. The data sets were aligned to the virtual CAD model. Afterwards, the 3D differences were quantitatively and qualitatively analyzed.

\section{Impression making}

Individualized custom trays divided into small compartments with pattern resin (Palavit G, Heraeus Kulzer, Hanau, Germany) or, if requested by the manufacturer, individual light-curing resin trays (Triad VLC Löffelmaterial, Heraeus Kulzer, Hanau, Germany), were used as impression trays.

Wire rests (for most impressions, two short straight pieces of orthodontic wire laid across the impression tray parallel to one another in vestibular-oral direction) or an adjustable mount to which the steel master model was attached at its base and then lowered into the compartment holding the impression material (for diagnostic cast impressions) ensured that the master model and the preparation margin were fully covered with impression material but could not sink deeply into the impression material under its own weight. Proper tray adhesives (Table 1b) were applied in a thin layer, which was allowed to dry for $10 \mathrm{~min}$.

For monophase impressions, the tray was first filled with the respective impression material (Table 1a) and then the master model was covered with the same material from an application syringe in an upward spiral movement, starting at the chamfer. For one-stage two-phase impressions, the tray was first filled with the higher viscosity material (Table 1a) using an automatic mixing unit (Table 1b), and afterwards the master model was covered with the lower viscosity material (Table 1a) with the helical movement starting at the chamfer. An automix syringe was used for the lower viscosity materials. The covered master model was lowered into the tray material until the chamfer was completely covered or the wire rests were touched. The master model remained in the impression without applying any pressure for a time span according to the manufacturer's instructions plus an extra $3 \mathrm{~min}$ with regard to room temperature (instead of body temperature).

The two-phase putty and wash tray material was 


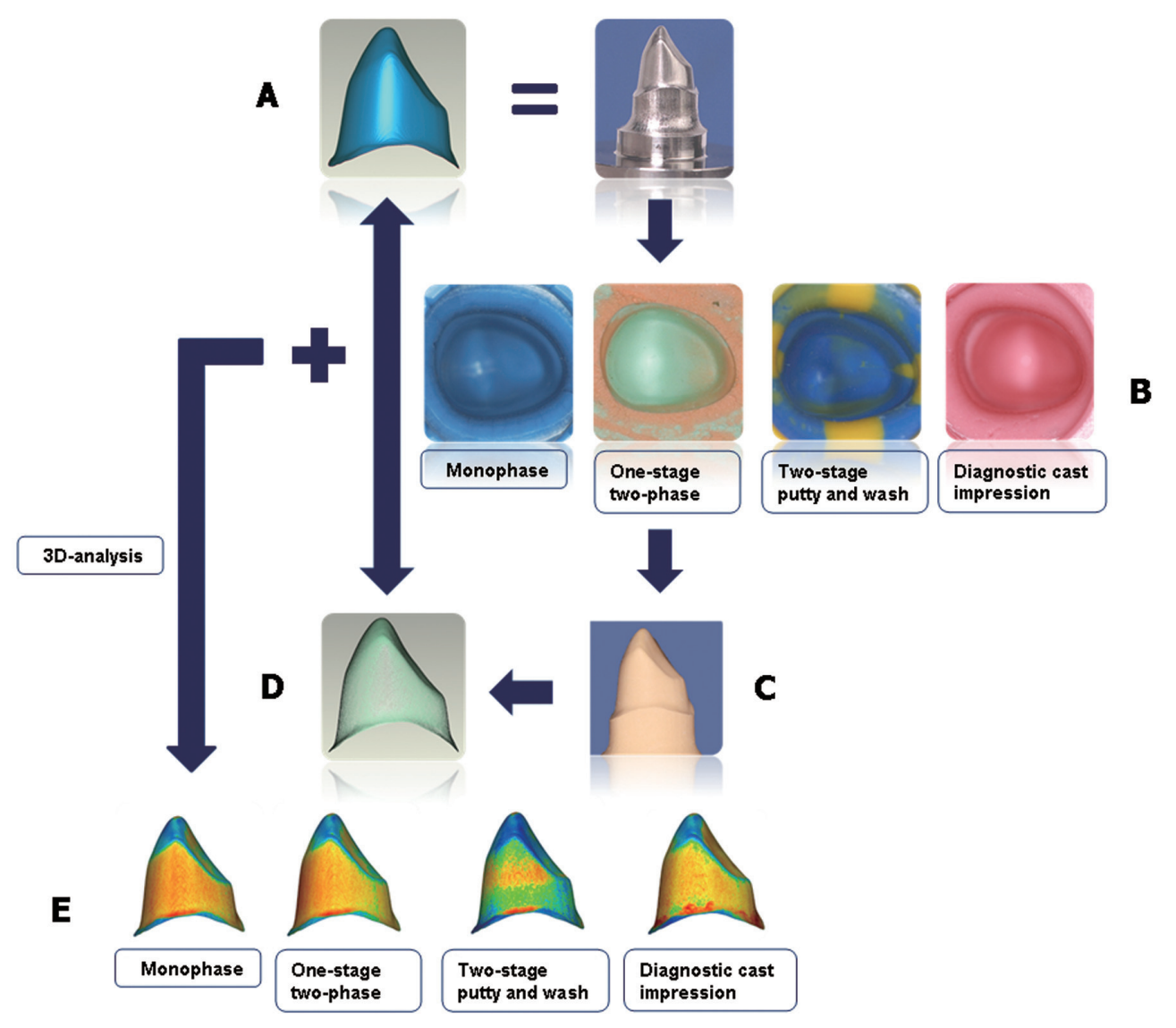

Fig. 1 Evaluation procedure based on a master model and virtual CAD model (A) for quantitative and qualitative $3 \mathrm{D}$ analysis.

Duplicate gypsum dies (C) are made from different impression materials/techniques (B). The digital data of the duplicate gypsum dies (D) are aligned to the virtual CAD model (A) before 3D differences are calculated (E).

mixed either with a mixing unit or by hand (according to the manufacturer's instructions) and filled into the prepared tray. After complete setting of the putty material, the master model was removed from the preliminary impression and checked for flaws. The material was cut back to the tray's rim. Four grooves were cut crosswise into the putty and the preparation margin was circularly removed. For the wash impression, the master model was covered as described before and reinserted into the preliminary impression.

Impressions with materials for diagnostic casts (Table 1a) were either automixed (Table 1b) or hand mixed. The powder/water ratio for the irreversible hydrocolloids was measured according to the manufacturer's instructions (9 g powder: $17.5 \mathrm{~mL}$ water). First, the powder was added to the mixing bowl and afterwards the water. A spatula was used to blend powder and water together for several seconds until moist.

The automix device was turned on. Holding the spatula firmly and positioning it at the top of the mixing bowl, it was moved at a straight line down to the center of the bowl twice. The device was stopped, the spatula was cleaned at the rim of the bowl and the downward movement was repeated after turning the device on again, resulting in a machine mixing time of $15 \mathrm{~s}$. The hand-mixed hydrocolloid was well stirred for $40 \mathrm{~s}$ until a homogeneous consistency was reached. The impression material was then filled into the tray compartment.

An addition-curing silicone for diagnostic cast impressions (Table 1a) was also mixed with a mixing unit (Table 1b).

All impressions were checked for flaws after removing the master model using $2.5 \times$ magnification glasses.

\section{Manufacturing of duplicate gypsum dies}

Impressions rested for $4 \mathrm{~h}$ after removal, to achieve optimal elastic recovery. The only exceptions were the reversible hydrocolloid precision impressions and the irreversible hydrocolloid diagnostic cast impressions, which were poured immediately after removing the steel master die. The impressions were poured a single time with class 4 gypsum (Esthetic-rock, Dentona DENTAL, 
Table 1a Impression materials; number in this study, manufacturers, and LOT numbers

\begin{tabular}{lll}
\hline No. Material & Material Type & $\begin{array}{l}\text { Manufacturer } \\
\text { (all Germany) }\end{array}$ \\
\hline
\end{tabular}

1 Dimension Garant L

2 Dimension Garant L Quick $\quad$ Light body VPS

\section{Dimension Penta $\mathrm{H}$}

\section{Dimension Penta H Quick Putty}

\begin{tabular}{|c|c|c|}
\hline 5 & Express Penta Putty & \\
\hline 6 & Express STD & \\
\hline 7 & Impregum Garant L DuoSoft & Light-body PE \\
\hline 8 & Impregum Penta & \multirow{2}{*}{ Heavy-body PE } \\
\hline 9 & Impregum Penta Soft & \\
\hline 10 & Imp. Penta Soft Quick Medium Body & Medium-body VPS \\
\hline 11 & Impregum L DuoSoft Quick & Light-body PE \\
\hline 12 & Impregum Penta H DuoSoft Quick base & Heavy-body PE \\
\hline 13 & $\begin{array}{l}\text { Impregum Penta H DuoSoft Quick } \\
\text { catalyst }\end{array}$ & $\begin{array}{c}\text { Catalyst for heavy-body } \\
\text { PE }\end{array}$ \\
\hline 14 & Korsil & Heavy-body VPS \\
\hline 15 & Palgat plus quick & Irreversible hydrocolloid \\
\hline 16 & Honigum Light & Light-body VPS \\
\hline 17 & Honigum MixStar Heavy & \multirow{4}{*}{ Heavy-body VPS } \\
\hline 18 & Status blue & \\
\hline 19 & Flexitime Dynamix Putty & \\
\hline 20 & Flexitime Fast\&Scan Dynamix Putty & \\
\hline 21 & Flexitime Fast\&Scan Light Flow & Light-body VPS \\
\hline 22 & Flexitime Fast\&Scan Medium Flow & Medium-body VPS \\
\hline 23 & Flexitime Light Flow & Light-body VPS \\
\hline 24 & Flexitime Medium Flow & Medium-body VPS \\
\hline 25 & Optosil P plus & Heavy-body CS \\
\hline 26 & Xantopren VL plus & Light-body CS \\
\hline 27 & Identium Heavy & Heavy-body VPS \\
\hline 28 & Identium Medium & Medium-body VPS \\
\hline 29 & Identium Light & \multirow{2}{*}{ Light-body VPS } \\
\hline 30 & Panasil binetics contact $\mathrm{x}$-light & \\
\hline 31 & Panasil binetics putty fast & Heavy-body VPS \\
\hline
\end{tabular}

LOT No.

FW0067062/FW0063829/ FW0066930

FW0065084/FW0067062/ FW0059154/FW0065065

FW0061862/FW0058500/ FW0065699/103806

FW0064055/FW0061225/ FW0058595/FW0065825

3M ESPE,

Seefeld

165304

\begin{tabular}{c}
\hline B0004 2001-12/C0003 2001-12 \\
\hline FW0061722/0066761/107638 \\
\hline FW0061531/FW0061532 \\
\hline 180453 \\
\hline $174999 /$ B194744/195967 \\
\hline $196971 / 205895$ \\
\hline
\end{tabular}

\begin{tabular}{c}
\hline 163388 \\
\hline Z0010673
\end{tabular}

DMG,

Hamburg

$\begin{array}{r}615401 \\ \hline 615614 \\ \hline 340104 \\ \hline 330446 \\ \hline 330142 \\ \hline 330137 \\ \hline 330034 \\ \hline 330410 \\ \hline 180137 \\ \hline 190097 \\ \hline 110201-35 \\ \hline 110181-59 \\ \hline 110071 \\ \hline 110291-28 \\ \hline 110621-185 \\ \hline\end{array}$


Table 1b Adhesive liquids; manufacturers, LOT numbers, and mixing units. For material numbers please refer to Table 1a

\begin{tabular}{lcrc}
\hline \multicolumn{1}{c}{ Tray adhesives } & Used for materials & Manufacturer & LOT No. \\
\hline Polyether Adhesive & $8-9,12$ & 3M ESPE, Seefeld & FW0067100 \\
Universal Adhesive & $3-6,10,14,17-20,22,24,25$ & Heraeus Kulzer, Hanau & R1000325 \\
Identium Adhesive & 27,28 & Kettenbach, Eschenburg & R1100308 \\
Panasil Haftlack & 31 & Dentsply DeTrey, Konstanz \\
Fix Adhesive - Liquid & 15 & Manufacturer \\
\hline Mixing units & Used for materials & 3M ESPE, Seefeld \\
Pentamix 2 & $3-6,8-10,12,14,17,25$ & Heraeus Kulzer, Hanau \\
Pentamix 3 & 18 & Kettenbach, Eschenburg \\
Dynamix Mischgerät & $19,20,22,24$ & DUX Dental, Untrecht, The Netherlands \\
Sympress Dispenser & $27,28,31$ & 15 & \\
Alginator & 1598
\end{tabular}

Wipperfürth, Germany). The water/gypsum ratio was measured with digital scales and a measuring cup according to the manufacturer's instructions $(20 \mathrm{~mL}$ water with $100 \mathrm{~g}$ gypsum), with demineralized water being used and mechanical vacuum-mixing for $60 \mathrm{~s}$. Laboratory surfactants were not applied. After shortly rinsing the impressions with water, excess water was removed with a gentle stream of air, avoiding overdrying.

Four hours after pouring, the duplicate dies were removed and checked for flaws using the magnification glasses. Dies from final impressions that showed bubbles, voids, or those that were discolored were discarded. In the case of diagnostic cast impressions, bubbles and small gypsum pearls resulting from bubbles in the impression material were tolerated. Excessive gypsum material was removed with dry trimming only to avoid expansion after setting.

\section{$3 D$ analysis}

After digitizing, all measuring points clearly below the die's preparation margin — the parts of the die belonging to its base- were removed manually. The system software (ARGUS, Fraunhofer Institute for Applied Optics and Precision Engineering, Jena, Germany) was used for data handling. Outlier data points were removed (parameters: tolerance 200\%, minimum of 25 connected points) and an automatic filtering algorithm was applied (stray points removal with parameters: tolerance $400 \%$, kernel 4 and size 4 ). The file format for data export was *.asc (ASCII, American Standard Code for Information Interchange). This format can be read by a computer-aided design and analysis software (Surfacer 10.6, Imageware Inc., Ann Arbor, MI, USA). Data quality was further improved by using semiautomatic specific filtering algorithms for outliers and stray data points, which were iteratively developed (threshold, checking distance, planar or spherical filter) in previous studies ${ }^{3,9,53)}$. The procedure removes points from the selected point clouds that do not conform to local planar or spherical models that are specified by other points in their neighborhood. This function samples the data based on agreement with locally fit models. Two model types may be used: a planar model and a spherical model. For each point, the function computes the local neighborhood model and checks the distance between the point and the model. If the distance is larger than the specified tolerance distance, the point is identified as an outlier and is rejected. The threshold for the neighborhood size was set to $0.010 \mathrm{~mm}$ and the maximum deviation tolerated was $0.001 \mathrm{~mm}$. The cut back and filtered data sets afterwards consisted of about 60,000 measuring points each.

All digitized dies were oriented in a coordinate system of their own and had to be aligned to the virtual CAD model (best-fit registration) before a 3D analysis was possible (Surfacer and Geomagic Studio 9, Geomagic Inc., Research Triangle Park, NC, USA). The alignment or registration is a software algorithm calculation of the vector or the matrix for the three-dimensional movement of the digitized gypsum duplicate dies to the reference CAD model representing the steel master die. The calculation is based on the determination of the bestfit between each of the 60,000 data points per digitized gypsum die and the reference CAD model by computing the respective perpendicular to the reference CAD surface. The calculation was performed with a tolerance setting of $0.0001 \mathrm{~mm}$. Alignment precision was recorded as root mean square (RMS) error.

After the best possible alignment between each duplicate die and the reference CAD model had been reached, the calculation of differences was done by using the $3 \mathrm{D}$ analysis feature of the CAD software. As output data, the computation provided quantitative 
Table 2 Product names, impression techniques, abbreviation used for each group of ten gypsum duplicate dies, and mean positive and negative deviations $[\mathrm{mm}]$ from the virtual CAD model

\begin{tabular}{|c|c|c|c|c|}
\hline Product name & Impression technique & Abbreviation & $\begin{array}{l}\text { Mean positive } \\
\text { deviation }[\mathrm{mm}]\end{array}$ & $\begin{array}{l}\text { Mean negative } \\
\text { deviation }[\mathrm{mm}]\end{array}$ \\
\hline Impregum Penta Soft & monophase (m) & pe-m-imps & $0.0145^{\mathrm{c}, \mathrm{d}, \mathrm{e}}$ & $-0.0135^{\mathrm{j}, \mathrm{k}, 1}$ \\
\hline Impregum Penta & monophase (m) & pe-m-imp & $0.0118^{\mathrm{b}, \mathrm{c}, \mathrm{d}}$ & $-0.0097^{\mathrm{j}, \mathrm{k}, \mathrm{l}, \mathrm{m}}$ \\
\hline $\begin{array}{l}\text { Impregum Penta Soft Quick Medium } \\
\text { Body }\end{array}$ & monophase (m) & pe-m-impsq & $0.0134^{\mathrm{b}, \mathrm{c}, \mathrm{d}, \mathrm{e}}$ & $-0.0131^{\mathrm{j}, \mathrm{k}, \mathrm{l}, \mathrm{m}}$ \\
\hline Identium Medium & monophase (m) & vpe-m-idenm & $0.0117^{b, c, d}$ & $-0.0081^{\mathrm{m}}$ \\
\hline Impregum Penta H/Garant L DuoSoft & one-stage two-phase (o) & pe-o-impduo & $0.0108^{b, c, d}$ & $-0.0120^{\mathrm{j}, \mathrm{k}, \mathrm{l}, \mathrm{m}}$ \\
\hline Dimension Penta H/Garant L & one-stage two-phase (o) & vps-o-dim & $0.0092^{\mathrm{b}}$ & $-0.0085^{1, \mathrm{~m}}$ \\
\hline Impregum Penta H/L DuoSoft Quick & one-stage two-phase (o) & pe-o-impduoq & $0.0119^{\mathrm{b}, \mathrm{c}, \mathrm{d}}$ & $-0.0124^{\mathrm{j}, \mathrm{k}, \mathrm{l}, \mathrm{m}}$ \\
\hline Systoloid & one-stage two-phase (o) & rh-o-sys & $0.0090^{\mathrm{b}}$ & $-0.0117^{\mathrm{j}, \mathrm{k}, \mathrm{l}, \mathrm{m}}$ \\
\hline Honigum Heavy/Light Body & one-stage two-phase (o) & vps-o-hon & $0.0111^{\mathrm{b}, \mathrm{c}, \mathrm{d}}$ & $-0.0115^{\mathrm{j}, \mathrm{k}, \mathrm{l}, \mathrm{m}}$ \\
\hline Identium Heavy/Light & one-stage two-phase (o) & vpe-o-idenhl & $0.0131^{\mathrm{b}, \mathrm{c}, \mathrm{d}, \mathrm{e}}$ & $-0.0081^{\mathrm{m}}$ \\
\hline Flexitime Dynamix Putty/Light Flow & one-stage two-phase (o) & vps-o-flexpl & $0.0103^{\mathrm{b}, \mathrm{c}}$ & $-0.0089^{\mathrm{k}, 1, \mathrm{~m}}$ \\
\hline Flexitime Dynamix Putty/Medium Flow & one-stage two-phase (o) & vps-o-flexpm & $0.0145^{\mathrm{c}, \mathrm{d}, \mathrm{e}}$ & $-0.0098^{\mathrm{j}, \mathrm{k}, \mathrm{l}, \mathrm{m}}$ \\
\hline Flexitime Fast und Scan Light Flow & one-stage two-phase (o) & vps-o-flexl & $0.0103^{\mathrm{b}, \mathrm{c}}$ & $-0.0087^{1, \mathrm{~m}}$ \\
\hline Flexitime Fast und Scan Medium Flow & one-stage two-phase (o) & vps-o-flexm & $0.0092^{\mathrm{b}}$ & $-0.0085^{1, \mathrm{~m}}$ \\
\hline Palgat Plus manual & diagnotic cast impression (d) & ih-d-pal-man & $0.0165^{\mathrm{e}}$ & $-0.0099^{\mathrm{j}, \mathrm{k}, \mathrm{l}, \mathrm{m}}$ \\
\hline Palgat Plus automatic & diagnotic cast impression (d) & ih-d-pal-aut & 0.0169 e & $-0.0100^{\mathrm{j}, \mathrm{k}, 1, \mathrm{~m}}$ \\
\hline Status Blue & diagnotic cast impression (d) & vps-d-blue & $0.0118^{\mathrm{b}, \mathrm{c}, \mathrm{d}}$ & $-0.0145^{j}$ \\
\hline Dimension Penta H/Garant L Quick & two-stage putty and wash (t) & vps-t-dimq & $0.0017^{\mathrm{a}}$ & $-0.0625^{\mathrm{g}}$ \\
\hline Korsil (experimental material) & two-stage putty and wash (t) & vps-t-em & $0.0100^{\mathrm{b}, \mathrm{c}}$ & $-0.0366^{\mathrm{h}}$ \\
\hline Express Penta Putty & two-stage putty and wash (t) & vps-t-expp & $0.0154^{\mathrm{d}, \mathrm{e}}$ & $-0.0186^{\mathrm{i}}$ \\
\hline Optosil P plus/Xantopren VL plus & two-stage putty and wash (t) & cs-t-opt & $0.0318^{f}$ & $-0.0121^{\mathrm{j}, \mathrm{k}, \mathrm{l}, \mathrm{m}}$ \\
\hline Express STD & two-stage putty and wash (t) & vps-t-exps & $0.0345^{\mathrm{f}}$ & $-0.0141^{\mathrm{j}, \mathrm{k}}$ \\
\hline Honigum Heavy/Light Body & two-stage putty and wash (t) & vps-t-hon & $0.0106^{\mathrm{b}, \mathrm{c}}$ & $-0.0102^{\mathrm{j}, \mathrm{k}, \mathrm{l}, \mathrm{m}}$ \\
\hline $\begin{array}{l}\text { Panasil binetics putty fast/ } \\
\text { initial contact } \mathrm{x} \text {-light }\end{array}$ & two-stage putty and wash (t) & vpe-t-pan & $0.0110^{\mathrm{b}, \mathrm{c}, \mathrm{d}}$ & $-0.0107^{\mathrm{j}, \mathrm{k}, \mathrm{l}, \mathrm{m}}$ \\
\hline
\end{tabular}

Letters in superscript indicate deviations belonging to one or more of six (positive: $a-f)$ or seven (negative: $g-m)$ different homogeneous subgroups identified with the Student-Newman-Keuls post hoc algorithm. Values with different letters are significantly different from one another, while values indexed with the same letter do not show any significant difference.

data (maximum positive deviations, mean positive deviations, mean negative deviations, maximum negative deviations, and a frequency distribution vertical-bar graph) as well as qualitative data in the form of color-coded graphs (color difference map), the latter showing the distribution of the $3 \mathrm{D}$ deviations over the complete surface of each gypsum die.

For better comparability, data were sampled to an even measurement point distance of $50 \mu \mathrm{m}$, resulting in $23,650 \pm 370$ points to be analyzed per die. A perpendicular, the smallest distance from each measurement point to the virtual CAD model, was calculated using the CAD software. All points lying outside or above the CAD-models' surface were defined as positive deviations, while those lying inside or below this surface were defined as negative deviations. The values obtained for a series of 10 duplicate dies are reported as "mean positive deviation" and "mean negative deviation" respectively. In order to assess the complete $3 \mathrm{D}$ information and in addition to the 
quantitative analysis, a qualitative analysis using colorcoded graphs was performed. The ten data sets of each test series were added to one single data set for the calculation of the color graphs.

\section{Statistical analysis}

For comparison of the different impression materials and techniques, the median, minima, maxima, and the interquartile range of the mean positive and negative deviations are shown in box-and-whisker plots. One-way analysis of variance was used for statistical analysis at a level of significance of $\alpha=0.05$ (SPSS 20.0, IBM Corporation, Armonk, NY, USA). The Levene test for homogeneity of variances and the Student-NewmanKeuls (SNK) post hoc test algorithm for equal variance were applied.

\section{RESULTS}

In order to clearly differentiate between gypsum duplicate dies which are reduced in circumference or height and those dies which are enlarged in either dimension, positive and negative values will be reported separately in the following. While the negative most value would be mathematically correct be referred to as the minimum value, those values will be reported as "maximum negative" with regard to their clinical relevance and the later discussion.

The range of RMS error for monophase (4 groups), one-stage two-phase (10 groups), two-stage putty and wash (7 groups), and diagnostic cast impressions (3 groups) were 8.0-22.9 $\mu \mathrm{m}$ (mean for all monophase impressions: $15.8 \mu \mathrm{m}$, SD 5.4), 9.3-15.2 $\mu \mathrm{m}$ (mean for all one-stage two-phase impressions: $12.1 \mu \mathrm{m}$, SD 2.1), 11.9-39.0 $\mu \mathrm{m}$ (mean for all two-stage putty and wash impressions: $23.9 \mu \mathrm{m}, \mathrm{SD} 10.3$ ), and 13.8-14.8 $\mu \mathrm{m}$ (mean for all diagnostic cast materials/mixing techniques: 14.2 $\mu \mathrm{m}, \mathrm{SD} 0.5)$, respectively.

Depending on the impression technique (without differentiation between the impression materials), the three-dimensional positive and negative mean deviations of all values for the monophase technique were $+12.9 /-11.1 \mu \mathrm{m}$ (SD 3.4/3.6) and 3D maximum positive and negative deviation values were $+83.6 /-88.7$ $\mu \mathrm{m}$. For the one-stage two-phase technique, the 3D positive and negative mean deviation values of all impressions were $+10.9 /-10.0 \mu \mathrm{m}$ (SD 2.8/2.3) and 3D maximum positive and negative deviation values of $+207.3 /-129.9 \mu \mathrm{m}$ were found. The pooled two-stage putty and wash technique impressions showed mean positive and negative values of $+16.5 /-23.5 \mu \mathrm{m}$ (SD $11.8 / 18.8$ ) and maximum positive and negative values of $+196.5 /-154.9 \mu \mathrm{m}$. All of the diagnostic cast materials together resulted in mean positive and negative values of $+15.0 /-11.5 \mu \mathrm{m}$ (SD 4.9/3.4), and maximum positive and negative values of $+257.0 /-20.6 \mu \mathrm{m}$.

The distribution of the deviations on the die surface is shown in color-coded graphs. Monophase impressions (Fig. 2: A), one-stage two-phase impressions (Fig. 2: B), and irreversible hydrocolloids for diagnostic cast impressions (Fig. 2: D) showed a circumferentially slightly enlarged representation of the master model and a rounding of edges. The dies were enlarged in height. Gypsum pearls resulting from bubbles can be found in irreversible hydrocolloids (darker red dots). Two-stage putty and wash impressions (Fig. 2: C) showed an elongation and a circumferential reduction. The latter was also found for addition-curing silicone for diagnostic cast impressions (Fig. 3: C).

Figures 4 (monophase, one-stage two-phase

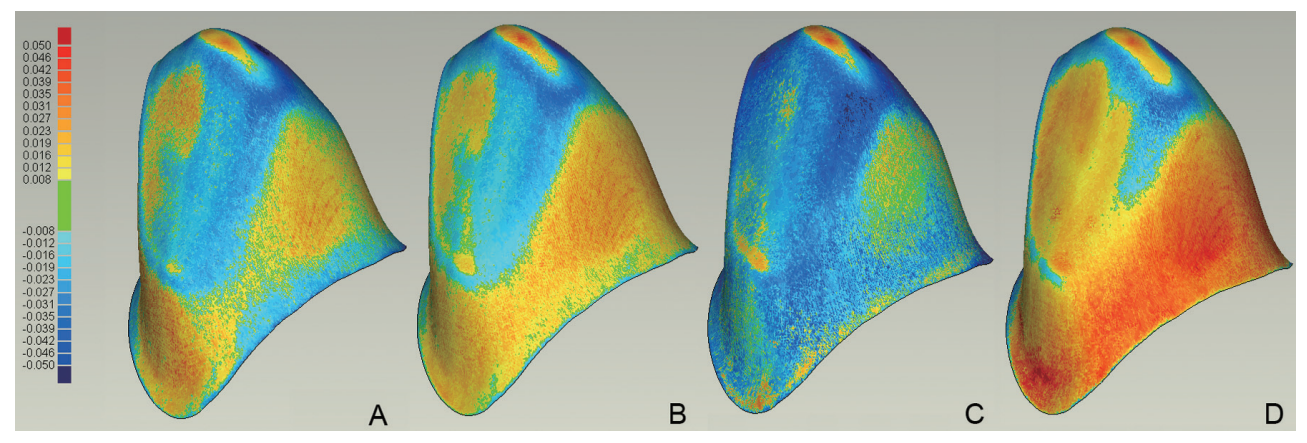

Fig. 2 Qualitative analysis; typical examples for monophase (A), one-stage two-phase (B), two-phase putty and wash (C), and diagnostic cast impressions (D), the latter made with irreversible hydrocolloid.

Yellow to red colors show enlargement, blue colors show reduction in size. The typical deviations for monophase (A) and one-stage two-phase (B) impressions were circumferential enlargement, an elongation of the tip and a rounding and thus reduction of edges. In addition to bubbles shown as dark red spots, these effects are more pronounced in diagnostic cast impressions with irreversible hydrocolloid (D). In contrast, two-phase putty and wash impressions clearly showed a generalized circumferential reduction alongside an enlargement in height. 


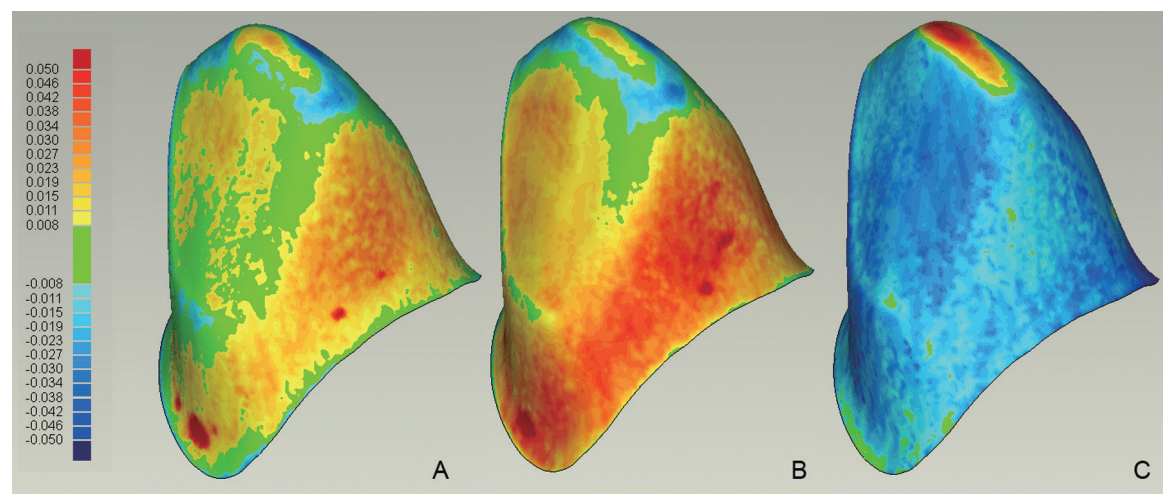

Fig. 3 Qualitative analysis; results for hand-(A) and machine- (B) mixed irreversible hydrocolloid in comparison to diagnostic cast impressions made with addition-curing silicone (C).

Yellow to red colors show enlargement, blue colors show reduction in size. The dark red spots represent gypsum pearls resulting from bubbles in the impression material. While hand- and machine-mixed irreversible hydrocolloid impressions for diagnostic casts (A and B) showed circumferential enlargement, elongation of the tip and rounding and thus reduction of edges similar to high precision monophase and one-stage two-phase impressions, the addition-curing silicone (C) for diagnostic casts showed a pronounced generalized circumferential reduction alongside an enlargement in height similar to some of the precision two-stage putty and wash impressions.

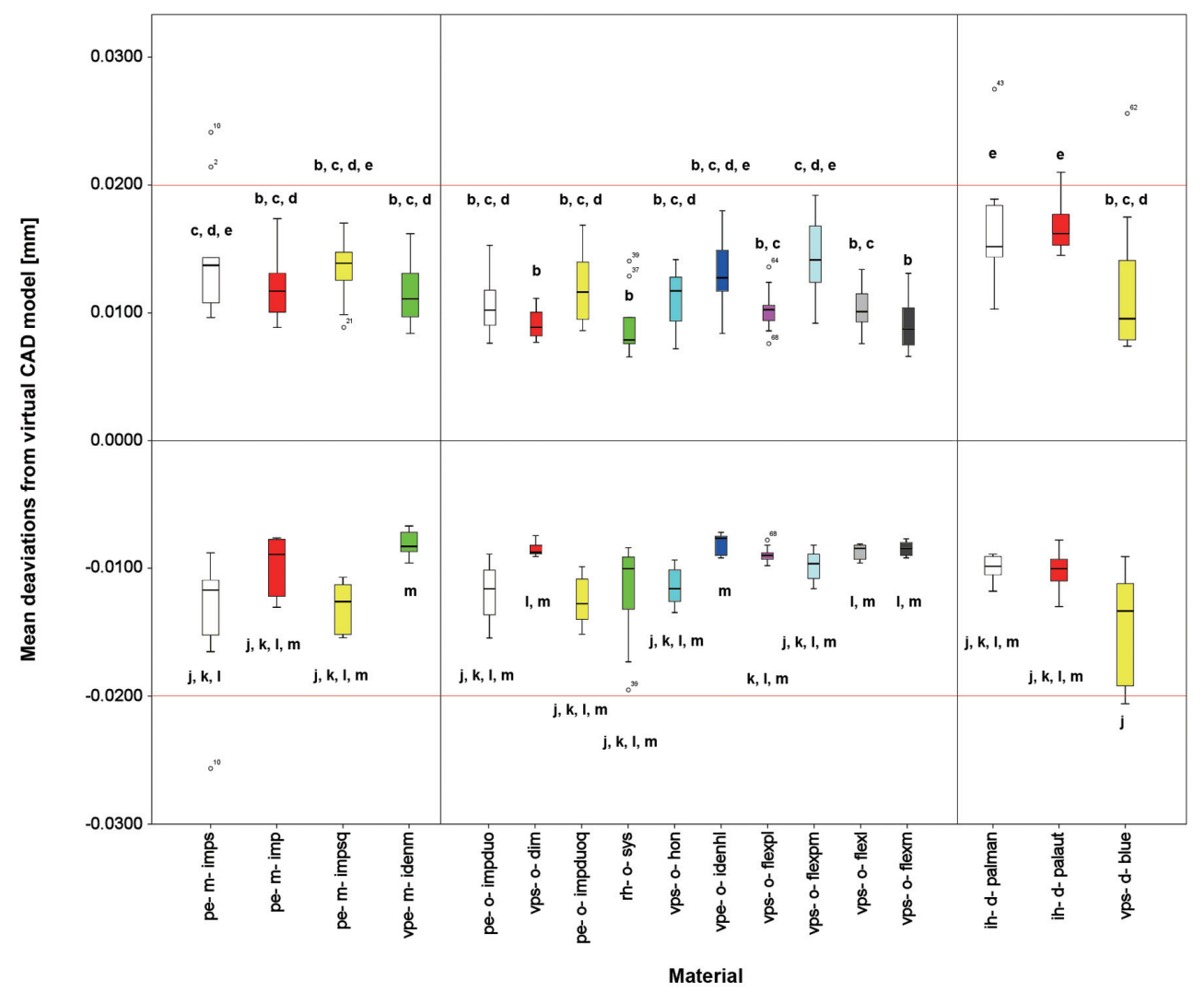

Fig. 4 Mean positive and negative deviations.

Group on the left: monophase (m) impression technique, middle: one-stage two-phase (o) impression technique, on the right: diagnostic cast impression materials (either vinyl polysiloxane (vps) or irreversible hydrocolloid (ih)). Benchmark was set to $\pm 0.02 \mathrm{~mm}$. Outlier values are more than 1.5 times the box width away from the boxes' outer boundaries and are indicated by circles. Boxes with different letters are significantly different from one another, while those indexed with the same letter do not show any significant difference. 
impression technique and diagnostic cast impressions) and 5 (two-stage putty and wash impression technique) as well as Table 2 (mean positive and negative deviations) show the results for the mean positive and negative deviations analyzed with one-way analysis of variance. For tests assuming equal variances such as the Student-Newman-Keuls (SNK) post hoc algorithm used here, homogeneous subsets can be constructed using a range determined by the specific test being used (Table 2). Six subgroups were determined for the mean positive deviations (a to f) and 7 subgroups ( $\mathrm{g}$ to $\mathrm{m}$ ) were found for the mean negative deviations by SNK.

Except for the only condensation-curing silicone tested in this study (cs-t-opt) and one of the vps materials (vps-t-exps), which both underwent the twostage putty and wash impression technique, all mean positive deviations were below the benchmark of $0.02 \mathrm{~mm}( \pm 20 \mu \mathrm{m})$. This also applies to the spread of values analyzing materials for final impressions when the monophase and one-stage two-phase impression technique were used, and for three of the seven material combinations that were used for the two-stage putty and wash impression technique. While the mean positive deviations of materials for diagnostic casts are also below the benchmark of $\pm 0.02 \mathrm{~mm}$, these materials either show more widespread values or deviations above the benchmark. The spread of values and large mean negative deviations are especially pronounced for the vps-based diagnostic cast impression material.

The analysis of materials for the two-stage putty and wash impression technique generally showed larger mean negative deviations (vps-t-dimq, vps-t-em, vpst-expp) or a wider spread of values and mean positive deviations above the benchmark of $\pm 0.02 \mathrm{~mm}$ (cs-t-opt and vps-t-exps, $p<0.000$ ). One material (vps-t-dimq) showed a particularly notable small mean positive deviation interlinked with larger mean negative deviations $(p<0.000)$. However, two material combinations (vpst-hon and vps-t-pan) showed results comparable to the other impressions techniques tested $(p=0.1197)$.

While the mean positive differences between subgroups b and e (Table 2) were statistically significant, the value range between 0.009 and 0.0169 $\mathrm{mm}(9-17 \mu \mathrm{m})$ was rather small. A similar range was found for the mean negative deviations of subgroups $\mathrm{j}-\mathrm{m}$ (Table 2). Again, differences were statistically significant $(p<0.000)$ but showed a small range with mean negative values between -0.0145 and $-0.0081 \mathrm{~mm}$ $(-15$ to $-8 \mu \mathrm{m})$. Only three material combinations used for the two-stage putty and wash impression technique showed significantly higher mean negative values when compared with subgroups j-m: vps-t-dimq (subgroup g), vps-t-em (subgroup h), and vps-t-expp (subgroup i).

\section{DISCUSSION}

Three-dimensional analysis is based on the comparison of digital data (e.g., duplicate dies) to a master form. Computer-aided alignment of measurement data and master form allows for overcoming limitations of the method described by Yamashita et al. ${ }^{54)}$. As the experimental set-up used for this study had been developed in 1999 by Luthardt and was used ever since in his research group, it could be regarded as established. A variation of the set-up has been successfully used for the analysis of changes in gypsum dies over time ${ }^{9)}$.

As the set-up always includes gypsum expansion and digitizing error, a high-precision resin-modified, class 4 gypsum as well as a high-precision digitizing system with a low measurement uncertainty had to be used. Following the originally developed standard operation procedure, data sets were reduced to an even measurement point distance of $50 \mu \mathrm{m}$ in order to reduce any influence of a differing data density on the results. This procedure also allows for a comparison of data gathered with different digitalization systems that have an unequal resolution. As always the same digitalization system was used in the study presented here, the influence of the data density is circumstantial. The data reduction also shortened the calculation time, which was an important factor a decade ago due to the performance of the computers used at that time. As even common personal computers nowadays are suitable for running the $3 \mathrm{D}$ analysis software and calculating results in a reasonably short time, one could argue that this step is not necessary any longer for study designs involving only a single digitizing system.

The precision of the data alignment before analysis, which was determined by the calculation of the RMSerror, is also of crucial significance for the systematic error. The precision of the alignment is in accordance with or even lower than previously reported ${ }^{8,55,56)}$. The RMS-error always combines the systematic error of the experimental setup including the measurement uncertainty of the digitizing system used and the deviations between duplicate die and master CAD-model themselves. For the RMS-error, all positive and negative values are first squared, then the mean is calculated and finally the root is extracted thus preventing positive and negative values to neutralize each other. In this study, a RMS-error of $8 \mu \mathrm{m}$ represents a series of dies without noteworthy deviations to the CAD-model or deviations within the systematic error of the experimental setup. Accordingly, 3D differences below $\pm 8 \mu \mathrm{m}$ cannot be detected using the presented method. A high RMS-error suggests larger deviations, given the fact that there is a low systematic error. A high RMS-error in combination with a high standard deviation is a sign for considerable variation between groups of dies made with the same impressions technique, as is the case for the two-phase putty and wash data presented here.

While, in theory, mechanical digitizing systems show a lower measurement uncertainty, such systems have limitations where free form surfaces are to be measured. Free form surfaces are surfaces with complex geometry such as prepared teeth. Thus, the systematic error cannot be lowered by using a mechanical digitizing system $^{13,36)}$. An alternative to high-precision optical digitizing systems could be the use of $\mu-\mathrm{CT}^{7}$. The access to such systems is generally limited and investment 
costs would be comparatively high.

The three-dimensional analysis of the deviations between the virtual CAD-model, which is the digital representation of the steel master model, and the digital data of the gypsum dies renders the maximum positive, maximum negative, mean positive, and mean negative deviations between each gypsum die and the steel master model. Using the CAD software, the distance between every single of the above mentioned approximately 23,500 measuring points per gypsum die and the CADmodel can be calculated. Due to the small distance of $50 \mu \mathrm{m}$ between these points, the free-form shape of the prepared die (its surface) is fully described. The reduction to single linear two-dimensional measurements between predefined corresponding measuring points and the immanent loss of 3D information can thus be overcome. Also, there is no need for a shape restriction when freeform surfaces can be analyzed just as well as clearly geometrically defined shapes such as cones or cylinders. The assessment of many different experimental set-ups close to the dental clinical setting hence becomes possible $2-4,8,37,45-47,49,57,58)$.

Based on the experience from former in vitro and in vivo studies ${ }^{3,9,45-48,53,57)}$, maximum positive and negative deviations, which are also calculated by the CAD software, were not further evaluated. These values are usually the result of single or very few outlier points and are of little informative value. Positive and negative deviations of the duplicate dies from the virtual CADmodel can be evaluated only in relation to the latter. Positive values represent a duplicate die which is enlarged in circumference and/or height while negative values show a reduced circumference or shortening in relation to the master CAD-model. If there is only an enlargement found in all areas of the die (circumference, edges and height), negative values will be a result of the measuring uncertainty of the digitizing system used and will thus show values only equal to or below this magnitude. In contrary, negative deviations above the measuring uncertainty of the digitizing system indicate a reduction of circumference and/or height as well as a rounding of edges. These values must be reported separately in order to clearly differentiate between enlargement and reduction. In a combined presentation of both, positive and negative values, this could be a major confounder of the results. For example a die being clearly reduced in height and somewhat enlarged in circumference will show close to none deviation in the quantitative evaluation, if positive and negative values were combined.

In a study on the influence of laboratory surfactant by Tredwin et al., different type 4 dental stones and impression materials were used than in the study at hand ${ }^{33)}$. However, a possible negative influence of a laboratory surfactant on the achievable precision could not be ruled out. For this reason, the use of surfactant was discarded. Using the wire rests and the stand prevented the materials from being distorted by the steel master model, especially during the critical phase towards the end of setting.

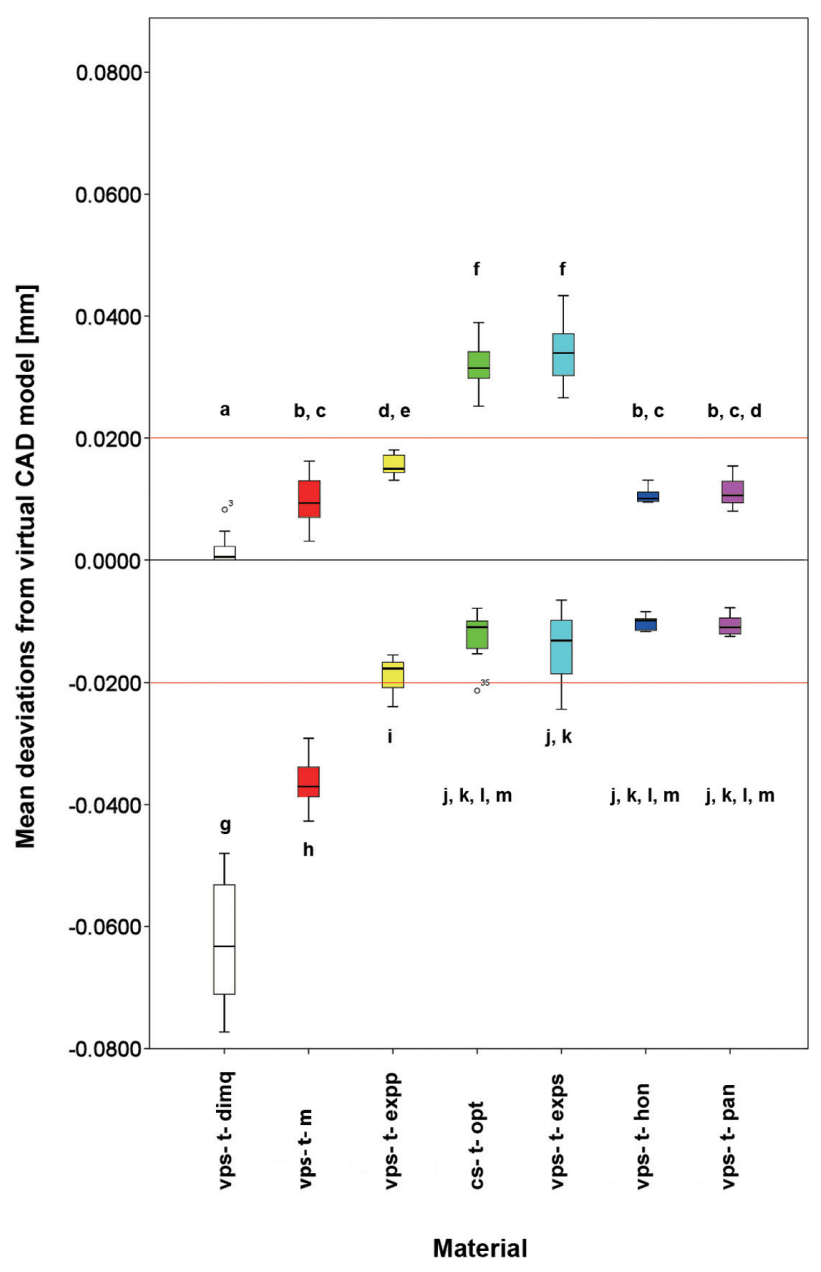

Fig. 5 Mean positive and negative deviations of the duplicate dies to the virtual master model (twostage putty and wash technique (t)).

Benchmark was set to $\pm 0.02 \mathrm{~mm}$. Note the different scale of the y-axis compared with Fig. $4: \pm 0.08 \mathrm{~mm}$ $( \pm 80 \mu \mathrm{m})$ instead of $\pm 0.03 \mathrm{~mm}( \pm 30 \mu \mathrm{m})$. Outlier values are more than 1.5 times the box width away from the boxes' outer boundaries and are indicated by circles. Boxes with different letters are significantly different from one another, while those indexed with the same letter do not show any significant difference.

While summing up the positive and negative mean deviations found by the quantitative evaluation and presenting them in box-and-whisker plots (Figs. 4 and 5) is especially convenient for a benchmarking of many different impression techniques and materials, the complete information can only be encompassed, if in addition to the quantitative analysis the qualitative information on the distribution of deviations over the die surface (Figs. 2 and 3) is taken into consideration. The combination of these results is one of the major advantages of this experimental procedure. Based on previous studies, 3D mean deviations of $\pm 0.02 \mathrm{~mm}$ for 
in vitro conditions and \pm 0.04 to $0.05 \mathrm{~mm}$ in clinical sites are to be expected for high-precision final impression materials and techniques ${ }^{27,47,53,58)}$.

The qualitative analysis (Figs. 2 and 3) shows the distribution of deviations on the prepared tooth's surface. For monophase and one-stage two-phase final impressions, a circumferential enlargement and a rounding of edges has shown to be typical, independently of the impression material and combination used. In contrast, the circumference of dies resulting from two-phase putty and wash impressions is reduced. Repositioning the tray with putty and wash material causes a certain distortion, which cannot be compensated by the material's ability for elastic recovery. This effect can be reduced depending on the flow and viscosity of the wash material ${ }^{28}$, which would give an explanation for the two materials with comparably smaller deviations despite the impression technique used (vps-t-hon and vps-t-pan).

Comparing the materials for diagnostic cast impressions (Fig. 3), hand- and machine-mixed irreversible hydrocolloid show a distribution of deviations similar to final impression materials (techniques $\mathrm{m}$ and o). This high precision was also found in a $3 \mathrm{D}$ analysis by Chandran et al. ${ }^{36)}$. Machine-mixing induced fewer and smaller bubbles and thus resulted in a better surface quality of the gypsum die. The vps-based material showed a distinct circumferential reduction in size, which could cause problems, if the impression itself or the resulting gypsum model was used for making provisional restorations or mouth guards, as examples.

Although statistically significantly different, the small range of mean values of $\pm 7-8 \mu \mathrm{m}$ between most subgroups, especially within the one-stage twophase impression materials, suggests a minor clinical relevance. The largest differences were found within the group of materials used for the two-stage putty and wash technique, which is in line with results achieved by Fenske $^{23)}$. The use of a die spacer for impressions rendering a reduced circumference is recommendable. Information on whether or not to use a die spacer has to be transmitted to the dental laboratory to achieve high precision.

The impression technique was shown to have a major influence on the achievable precision of duplicate dies independent of the composition of the materials used.

\section{CONCLUSION}

Three-dimensional analysis can give a complete picture of the precision of gypsum duplicate dies depending on the impression materials and impression techniques used. The visualization provides a detailed insight into occurring deviations in comparison to conventional in vitro analyses.

Processing aspects (manually or auto-mixing) and impression technique significantly influence the die precision. Independent of the materials used, very good $3 \mathrm{D}$ representation of the master die was found for the one-stage two-phase impression technique. However, using the two-stage putty and wash technique, only two out of six addition-curing vinyl polysiloxanes showed comparably good results.

\section{ACKNOWLEDGMENTS}

The German dental companies 3M Deutschland GmbH, Seefeld; DMG Chemisch-Pharmazeutische Fabrik $\mathrm{GmbH}$, Hamburg; Heraeus Kulzer GmbH, Hanau, and Kettenbach GmbH \& Co. KG, Eschenburg supported parts of this study.

Some of the results have been presented on the Academy of Dental Materials Annual Meeting, October 2-4 2008 in Würzburg, Germany; on the IADR/AADR/ CADR 83nd General Session, March 9-12 2004 in Baltimore, MD, USA and on the 41st AADR Annual Meeting, March 21-24 2012 in Tampa, FL, USA.

\section{REFERENCES}

1) Dastane A, Vaidyanathan TK, Vaidyanathan J, Mehra R, Hesby R. Development and evaluation of a new 3-D digitization and computer graphic system to study the anatomic tissue and restoration surfaces. J Oral Rehabil 1996; 23: 25-34.

2) DeLong R, Knorr S, Anderson GC, Hodges J, Pintado MR. Accuracy of contacts calculated from 3D images of occlusal surfaces. J Dent 2007; 35: 528-534.

3) Rudolph H, Luthardt RG, Walter MH. Computer-aided analysis of the influence of digitizing and surfacing on the accuracy in dental CAD/CAM technology. Comput Biol Med 2007; 37: 579-587.

4) Stimmelmayr M, Guth JF, Erdelt K, Edelhoff D, Beuer F. Digital evaluation of the reproducibility of implant scanbody fit-an in vitro study. Clin Oral Investig 2012; 16: 851-856.

5) White AJ, Fallis DW, Vandewalle KS. Analysis of intraarch and interarch measurements from digital models with 2 impression materials and a modeling process based on cone-beam computed tomography. Am J Orthod Dentofacial Orthop 2010; 137: 456 e1-9; discussion -7.

6) Sjogren AP, Lindgren JE, Huggare JA. Orthodontic study cast analysis - reproducibility of recordings and agreement between conventional and 3D virtual measurements. J Digit Imaging 2010; 23: 482-492.

7) Kamegawa M, Nakamura M, Fukui Y, Tsutsumi S, Hojo M. Direct 3-D morphological measurements of silicone rubber impression using micro-focus X-ray CT. Dent Mater J 2010; 29: 68-74.

8) Stimmelmayr M, Erdelt K, Guth JF, Happe A, Beuer F. Evaluation of impression accuracy for a four-implant mandibular model-a digital approach. Clin Oral Investig 2012; 16: 1137-1142.

9) Luthardt RG, Kuhmstedt P, Walter MH. A new method for the computer-aided evaluation of three-dimensional changes in gypsum materials. Dent Mater 2003; 19: 19-24.

10) Shah S, Sundaram G, Bartlett D, Sherriff M. The use of a 3D laser scanner using superimpositional software to assess the accuracy of impression techniques. J Dent 2004; 32: 653-658.

11) Schaefer O, Schmidt M, Goebel R, Kuepper H. Qualitative and quantitative three-dimensional accuracy of a single tooth captured by elastomeric impression materials: an in vitro study. J Prosthet Dent 2012; 108: 165-172.

12) Persson AS, Andersson M, Oden A, Sandborgh-Englund G. Computer aided analysis of digitized dental stone replicas by dental CAD/CAM technology. Dent Mater 2008; 24: 11231130.

13) Quaas S, Rudolph H, Luthardt RG. Direct mechanical data 
acquisition of dental impressions for the manufacturing of CAD/CAM restorations. J Dent 2007; 35: 903-908.

14) Chadwick RG, Mitchell HL, Ward S. A novel approach to evaluating the reproducibility of a replication technique for the manufacture of electroconductive replicas for use in quantitative clinical dental wear studies. J Oral Rehabil 2004; 31: 335-339

15) Wadhwani CP, Johnson GH, Lepe $X$, Raigrodski AJ. Accuracy of newly formulated fast-setting elastomeric impression materials. J Prosthet Dent 2005; 93: 530-539.

16) Hassan AK. Dimensional accuracy of 3 silicone dental impression materials. East Mediterr Health J 2006; 12: 632636.

17) Stober T, Johnson GH, Schmitter M. Accuracy of the newly formulated vinyl siloxanether elastomeric impression material. J Prosthet Dent 2010; 103: 228-239.

18) Levartovsky S, Levy G, Brosh T, Harel N, Ganor Y, Pilo R. Dimensional stability of polyvinyl siloxane impression material reproducing the sulcular area. Dent Mater J 2013; 32: 25-31.

19) Pereira JR, Murata KY, Valle AL, Ghizoni JS, Shiratori FK. Linear dimensional changes in plaster die models using different elastomeric materials. Braz Oral Res 2010; 24: 336341.

20) Alhouri N, Watts DC, McCord JF, Smith PW. Mathematical analysis of tooth and restoration contour using image analysis. Dent Mater 2004; 20: 893-899.

21) Faria AC, Rodrigues RC, Macedo AP, Mattos Mda G, Ribeiro $\mathrm{RF}$. Accuracy of stone casts obtained by different impression materials. Braz Oral Res 2008; 22: 293-298.

22) Al-Bakri IA, Hussey D, Al-Omari WM. The dimensional accuracy of four impression techniques with the use of addition silicone impression materials. J Clin Dent 2007; 18: 29-33.

23) Fenske $\mathrm{C}$. The influence of five impression techniques on the dimensional accuracy of master models. Braz Dent J 2000; 11: 19-27.

24) Franco EB, da Cunha LF, Herrera FS, Benetti AR. Accuracy of single-step versus 2-step double-mix impression technique. ISRN Dent 2011; 2011: 341546.

25) Gomez-Polo M, Celemin A, del Rio J, Sanchez A. Influence of technique and pouring time on dimensional stability of polyvinyl siloxane and polyether impressions. Int $\mathrm{J}$ Prosthodont 2012; 25: 353-356.

26) Nissan J, Laufer BZ, Brosh T, Assif D. Accuracy of three polyvinyl siloxane putty-wash impression techniques. J Prosthet Dent 2000; 83: 161-165.

27) Piwowarczyk A, Ottl P, Buchler A, Lauer HC, Hoffmann A. In vitro study on the dimensional accuracy of selected materials for monophase elastic impression making. Int J Prosthodont 2002; 15: 168-174.

28) Balkenhol M, Ferger P, Wostmann B. Dimensional accuracy of 2-stage putty-wash impressions: influence of impression trays and viscosity. Int J Prosthodont 2007; 20: 573-575.

29) Hoyos A, Soderholm KJ. Influence of tray rigidity and impression technique on accuracy of polyvinyl siloxane impressions. Int J Prosthodont 2011; 24: 49-54.

30) Nissan J, Gross M, Shifman A, Assif D. Effect of wash bulk on the accuracy of polyvinyl siloxane putty-wash impressions. $J$ Oral Rehabil 2002; 29: 357-361.

31) Omar R, Abdullah MA, Sherfudhin H. Influence on dimensional accuracy of volume of wash material introduced into pre-spaced putty/wash impressions. Eur J Prosthodont Restor Dent 2003; 11: 149-155.

32) Shiozawa M, Takahashi H, Finger WJ, Iwasaki N. Effects of the space for wash materials on sulcus depth reproduction with addition-curing silicone using two-step putty-wash technique. Dent Mater J 2013; 32: 150-155.

33) Tredwin CJ, Nesbit M, Butta R, Moles DR. Effect of a laboratory surfactant on compatibility of type IV dental stones with addition-cured silicone impression materials. Eur J Prosthodont Restor Dent 2008; 16: 73-76.

34) Brosky ME, Pesun IJ, Lowder PD, Delong R, Hodges JS. Laser digitization of casts to determine the effect of tray selection and cast formation technique on accuracy. J Prosthet Dent 2002; 87: 204-209.

35) Petrie CS, Walker MP, O'Mahony A M, Spencer P. Dimensional accuracy and surface detail reproduction of two hydrophilic vinyl polysiloxane impression materials tested under dry, moist, and wet conditions. J Prosthet Dent 2003; 90: $365-372$.

36) Chandran DT, Jagger DC, Jagger RG, Barbour ME. Two- and three-dimensional accuracy of dental impression materials: effects of storage time and moisture contamination. Biomed Mater Eng 2010; 20: 243-249.

37) Persson AS, Oden A, Andersson M, Sandborgh-Englund G. Digitization of simulated clinical dental impressions: virtual three-dimensional analysis of exactness. Dent Mater 2009; 25: 929-936.

38) Cayouette MJ, Burgess JO, Jones RE, Jr., Yuan CH. Three-dimensional analysis of dual-arch impression trays. Quintessence Int 2003; 34: 189-198.

39) Kwon JH, Son YH, Han CH, Kim S. Accuracy of implant impressions without impression copings: a three-dimensional analysis. J Prosthet Dent 2011; 105: 367-373.

40) Papaspyridakos P, Benic GI, Hogsett VL, White GS, Lal K, Gallucci GO. Accuracy of implant casts generated with splinted and non-splinted impression techniques for edentulous patients: an optical scanning study. Clin Oral Implants Res 2012; 23: 676-681.

41) Nassar U, Hussein B, Oko A, Carey JP, Flores-Mir C. Dimensional accuracy of 2 irreversible hydrocolloid alternative impression materials with immediate and delayed pouring. J Can Dent Assoc 2012; 78: c2.

42) Rodrigues SB, Augusto CR, Leitune VC, Samuel SM, Collares FM. Influence of delayed pouring on irreversible hydrocolloid properties. Braz Oral Res 2012; 26: 404-409.

43) Hamalian TA, Nasr E, Chidiac JJ. Impression materials in fixed prosthodontics: influence of choice on clinical procedure. J Prosthodont 2011; 20: 153-160.

44) Rubel BS. Impression materials: a comparative review of impression materials most commonly used in restorative dentistry. Dent Clin North Am 2007; 51: 629-642, vi.

45) Haim M, Luthardt RG, Rudolph $H$, Koch R, Walter MH, Quaas S. Randomized controlled clinical study on the accuracy of two-stage putty-and-wash impression materials. Int J Prosthodont 2009; 22: 296-302.

46) Luthardt RG, Walter MH, Weber A, Koch R, Rudolph H. Clinical parameters influencing the accuracy of 1- and 2-stage impressions: a randomized controlled trial. Int J Prosthodont 2008; 21: 322-327.

47) Rudolph H, Quaas S, Haim M, Preissler J, Walter MH, Koch $\mathrm{R}$, Luthardt RG. Randomized controlled clinical trial on the three-dimensional accuracy of fast-set impression materials. Clin Oral Investig 2013; 17: 1397-1406.

48) Rudolph H, Quaas S, Luthardt R. Precision of duplicate dies made with different impression making procedures. Dent Mater 2008; 25: e27-e28.

49) Ender A, Mehl A. Full arch scans: conventional versus digital impressions —an in-vitro study. Int J Comput Dent 2011; 14: 11-21.

50) Petrie CS, Walker MP, Theodotou N, Glaros AG, Williams K. Effect of measurement site on the dimensional accuracy of die-forming materials and techniques. Gen Dent 2004; 52: 228-232.

51) Hara S, Mitsugi M, Kanno T, Tatemoto Y. Computer-aided design provisionalization and implant insertion combined with optical scanning of plaster casts and computed 
tomography data. Ann Maxillofac Surg 2014; 4: 64-69.

52) Rudolph H, Salmen H, Quaas S, Luthardt RG. Comparison of different optical digitizing systems for dental CAD/CAM dataacquisition. J Dent Res 2009; 88 (Spec Iss B): 0373 (CED).

53) Luthardt RG, Koch R, Rudolph H, Walter MH. Qualitative computer aided evaluation of dental impressions in vivo. Dent Mater 2006; 22: 69-76.

54) Yamashita S, Ai M, Geng Q, Sato M, Shinoda H, Ando S. Application of a newly developed 3-D deformation measurement system to prosthetic dentistry. J Oral Rehabil 1996; 23: 849-855.

55) Del Corso M, Aba G, Vazquez L, Dargaud J, Dohan Ehrenfest DM. Optical three-dimensional scanning acquisition of the position of osseointegrated implants: an in vitro study to determine method accuracy and operational feasibility. Clin Implant Dent Relat Res 2009; 11: 214-221.

56) Mehl A, Ender A, Mormann W, Attin T. Accuracy testing of a new intraoral 3D camera. Int J Comput Dent 2009; 12: 1128.

57) Luthardt RG, Walter MH, Quaas S, Koch R, Rudolph H. Comparison of the three-dimensional correctness of impression techniques: a randomized controlled trial. Quintessence Int 2010; 41: 845-853.

58) Rudolph H, Rohl A, Walter MH, Luthardt RG, Quaas S. Performance of fast-setting impression materials in the reproduction of subgingival tooth surfaces without soft tissue retraction. Int J Prosthodont 2014; 27: 366-375. 